Historic, Archive Document

Do not assume content reflects current scientific knowledge, policies, or practices. 



\section{The Fremont Nursery}

\section{Price List of Fruit and Ornamental Trees, Vines, Small Fruits, Ect. SPRING, 1920}

\section{BY EXPRESS OR FREIGHT, PURCHASER PAYING CHARGES}

There is now in this country the greatest scarcity of fruit trees and small fruit plants that was ever known in the history of the business. This is caused by several reasons.

First, for a number years there was an over supply of stock and much of it was sold to the public at prices below the cost of production, even on the basis of prewar values, and much could not be sold at any price and was destroyed. As a consequence thousands of small growers throughout the country went out of business and some few of the larger ones went into bankruptcy.

Second, almost all of our seedlings for the production of fruit trees are impoi ? from France, and during the war the importation of them was so uncertain $t^{\prime}$ ' very few were imported and of those that were, many were ruined and worthless arrival owing to long delays, etc.

Third, labor has been scarce and high priced and it has been impossible for many firms to even attempt to grow their normal amount of stock, even if they had been able to obtain their supply of seedlings.

Fourth, peach seedlings are grown from seed in this country, but the cold $v_{1} 0$ of 1917 froze out the seed, which had been planted in the Fall, so that many I. $_{\text {. }}$ plowed up their entire planting in the Spring.

It takes several years after planting the seedling before the tree is ready for market, and therefore this coming shortage did not begin to be felt till last year. There is no hope for any improvement in most kinds of fruit trees for several years to come. The plantings of seedlings of stocks imported last Spring, after the close of the war, are for the most part failures, and at the present time no seedlings can be obtained for next season at all.

Some trees and plants, like peach trees and berry plants, which are grown entirely in this country, will gradually come into better supply in a year or two, but the outlook is very poor for any average supply of fruit trees for a number of years to come.

On the whole, there is probably less than 30 per cent of a normal supply of fruit trees in the country today, and far less than that percent of peach trees and grape and currant plants.

In ornamental stock the situation is not nearly as bad, but that is bound to become worse, owing to the absolute exclusion of everything in that line from foreign countries which went into effect June 1st by order of the U. S. Federal Horticultural Board.

In our price lists we always aim to avoid confusing you with all sorts of bewildering premium offers, usually designed to get rid of surplus varieties, and to make a plain statement of rock bottom prices for the best grade of stock on the market. That this plan is appreciated by our customers is evidenced by our constantly increasing trade.

To save you money or to pay you for any efforts among your neighbors, we will give you the benefit of the offer in the following paragraph:

$\$ 8$ ORDER: If your remittance amounts to $\$ 8$ you may select all stock at the 10 rate, regardless of the number ordered. Owing to the high cost of packing small orders, those for less than $\$ 8$ must be at the single rate unless the number ordered 
of a kind entitles you to a lower rate. We are glad to pack all small orders, the nature of which will allow them to be packed at a profit, but we reserve the right to refuse all or any part of an order (and return the money), the packing of which, owing to the nature of some certain part of the order, would consume all the profit on the order.

CLUB ORDERS-If the total remittance to us amounts to $\$ 25.00$ we will give you the benefit of the hundred rate on everything, regardless of the number ordered. If you do not want that amount, cannot you combine with your neighbors and make up an order of that much, and thus all get the benefit of the low rate? This will save you about one-third, besides it will reduce transportation charges proportionately. It will pay you well for your time to solicit orders from your locality at the single rate and get the trees at the hundred rate. We will put up each order separately, properly tagged, and ship all together.

NUMBER-Five trees of a kind, as tive apple or five peach, will be furnished at the 10 rate, and fifty at the 100 rate, five hundred at 1,000 rate. Trees of different kinds, as apple. peach, pear, etc. cannot be counted together to get the 100 rate, unless the total remittance amounts to $\$ 25.00$ as noted above.

TERMS-Cash or satisfactory reference. If you will send us good sound reference, we will ship your order and let you pay for it on receipt. We cannot send orders promiscuously without cash or reference.

SHIPPING DIRECTIONS-Always give full name and address and state how you wish your order shipped, whether by freight or express. We advise all small orders to be sent by express, as a small order often dries out if sent by freight and delayed a few days. Berry plants should always be sent by express. We can send small fruit plants and small sized trees by parcel post, but postage would have ${ }^{+} \mathrm{o}$ be added to the cost of stock.

QUANTITY-For large orders or for stock not offered here, send list of stock wanted for special tations.

\section{APPLE}

Each Per 10 Per 100

XX Grade, 5-7 ft,

First Jlass, 4-6 ft.

First Class, $4-5 \mathrm{ft}$.

\section{$\$ .75$}

.60

.40

$\$ 6.00$

5.00

3.50
DWARF APPLE

$\times$ ust Class, 3-4 ft.-_-_-_-_-_-_-_-_ $\$ .75$

First Class, 2-3 ft.-. 65

PEARS, STANDARD

XX Grade, 5-7 ft.

First Class, 4-6 ft.

First Class, 4-5 ft.

PEARS, DWAF

First Class, 3-4 ft._-_._-_-_-_ $\$ .75$

First Class, 2-3 ft. .75
.60

$$
\$ 500060
$$

Russian, $3-4 \mathrm{ft}$

$\$ 6.00$

5.00

$\$ 50.00$ 40.00

$\$ 7.50$

6.00

5.00

$\$ 60.00$

50.00

40.00

$\$ 6.00 \$ 45.00$

$5.00 \quad 35.00$

PEACHES

XX Grade, 5-7 ft.

First Class, 4-6 ft.-_-

First Class, 3-4 ft.___

First Class, 2-3 ft._- $40 \quad 3.50$

\section{CHERRIES, SWEET AND SOUR}

XX Grade, 5-6 ft._.

First Class, 4-5 ft.-_-_-___-_- $.75 \quad 6.00$

First Class, 3-4 ft._-_-_-_-_-_ $\quad .60 \quad 5.00$

Sour, 1 yr. XXX, about $3 \mathrm{ft}$

SWEET CHERRIES ON MAZZARD ROOT

XX Grade, 5-7 ft.

$\$ 1.25 \$ 10.00$

First Class, 4-6 ft._- $1.00 \quad 8.00$

\section{PLUMS}

XX Grade, 5-7 ft._. $\$ 1.25 \$ 10.00$

First Class, $4-6 \mathrm{ft} \quad-1.00-8.00$

First Class, 3-5 ft.

One year, 3-4 ft.

\section{QUINCES}

First Class, 3-4 ft.-_-_- $\$ .75 \$ 6.00$

First Class, 2-3 ft.

$\begin{array}{rr}.75 & \$ 6.00 \\ .65 & 5.50\end{array}$

Russian, 5-6 ft.
$\$ 50.00$

40.00

$\$ 45.00$

35.00

$$
\text { Other Leading Varieties, Fays, }
$$

Cherry, Wilder, etc.

GOOSEBERRY, 2 YEAR

Houghton _-

Downing -

Josselyn, Columbus and Chautauqua .50

\section{RASPBERRY}

Per 10 Per 100 Per 1000

$\$ 75.00$

60.00

45.00

45.00

$\$ 50.00$

40.00

3.50

25.00

$\$ 4.00 \$ 25.00$

20.00

$\$ 2.00 \$ \$ 15.00$

$2.50 \quad 20.00$

$4.00 \quad 30.00$

Cuthbert, Miller, St. Regis____ $\$ .50 \quad \$ 3.00 \quad \$ 25.00$

Cumberland, Gregg, Kansas _-_--- $\quad .50 \quad 3.00 \quad 25.00$

New Logan, King of Cliffs _-_._. $.60 \quad 3.50 \quad 28.00$

Columbian, Haymaker

\section{BLACKBERRY}

Snyder, Taylor, Erie $\$ .60 \$ 3.50$ 6.00
Each Per 10 Per 100

$\$ 4.50 \quad \$ 35.00$

$3.50 \quad 25.00$

7.50

$\$ 8.00 \quad \$ 50.00$

$6.00 \quad 40.00$

$6.00 \quad 45.00$

$6.00 \quad 40.00$

$8.00 \quad 60.00$

$7.00 \quad 55.00$

$5.00 \quad 45.00$

$\$ 2.50 \quad \$ 20.00$ 
Per 10 Per 100 Per 1000

Everbearing $\$ .40 \$ 2.00$ $\$ 15.00$ Senator Dunlap and Warfield_____- $.25 \quad 1.00$ Other varieties .25

6.00

8.00

\section{ROOTS}

Asparagus, 1 year $\$ .15$

Asparagus, 2 years .75

1.25

2.00

Rhubarb, 1 year, each

.10

$\$ 6.00$

7.00

6.00

\section{ORNAMENTAL TREES}

Each Per 10 Per 100

Alder, Imperial Cut-leaved, 8-10 ft._\$1.00

$\$ 9.00$

Crab, Bechtels Dbl. Flowering, 2-3

feet 1.00

Beech, purple Leaved, 4-6 ft._-_--- 1.25

Beech, Purple Leaved, 3-4 ft._-_-_ 1.00

Catalpa, Flowering, 5-6 $\mathrm{ft}_{\text {- }} .40$

Catalpa, Flowering, 6-8 ft._-___-_- .50

Catalpa, Flowering, 8-10 ft._-__-_-_ .75

Catalpa, Bungeii, straight stems

Elm, American, 6-8 ft._._._._____ $\quad .80$

Elm, American, 8-10 ft._.______-_ 1.00

Elm, American, 10-12 ft._.______-_ 1.25

Hawthorn, Red, white and Pink,

4-5 ft.

Horse-Chestnut, 5-6 ft._____ 1.00

Horse-Chestnut, 6-8 ft._-_-_-_-_-_-- 1.25

Magnolia, with ball of earth on roots, 3-4 ft.

Maple, Japan (Dwarf), 2-3 ft.---- 2.00

Maple, Ash-leaved, 6-8 ft._-___-_ .50

Maple, Ash-leaved, 8-10 ft._-___- .75

Maple, Norway, 5-6 ft._.___-_-_._. .40

Maple, Norway, 6-8 ft._____ 1.00

Maple, Norway, 8-10 ft._-______-_ 1.25

Maple, Norway, 10-12 ft._______- 1.50

Maple, Silver (Soft), 6-8 ft._-_-_ .60

Maple, Silver (Soft), 8-10 ft.-_-_-- .85

Maple, Sugar (Hard), 6-8 ft.____ .75

Maple, Sugar (Hard), 8-10 ft.-_--- 1.00

Maple, Schwedlers, 6-8 ft.______-_ 1.00

Maple, Schwedlers, 8-10 ft._-___- 1.50

Maple, Weir's Cut-leaved, 6-8 ft.-- .85

Maple, Weir's Cut-leaved, 8-10 ft.- 1.00

Mountain Ash, 6-8 ft._.-_._-_.-. .85

Poplar, Carolina, Lombardy and Norway, 5-6 ft. _-_ .30

Poplar, Carolina, Lombardy and Norway, 6-8 ft. -

Poplar, Carolina, Lombardy and Norway, 8-10 ft. -...---_-

Poplar, Carolina, Lombardy and

Norway, 10-12 ft.
Sycamore, Europ. (Oriental Plane), $6-8 \mathrm{ft}$.

Sycamore, Europ. (Oriental Plane), 8-10 ft. _. .90

Tulip Tree, 6-8 $\mathrm{ft}$.
9.00

3.50

4.00

6.00

10.00

7.00

7.50

10.00

9.00

8.00

11.00

10.00

12.00

5.00

7.50

6.00

10.00

10.00

15.00

7.50

9.00

7.50

2.50

4.00

5.00

6.00

6.00

7.50

9.00
$\$ 25.00$

35.00

50.00

70.00

90.00

60.00

85.00

100.00

40.00

50.00

50.00

80.00

\subsection{0}

30.00

40.00

50.00

50.00

60.00
Each Per 10 Per 100

Birch, Cut-leaved, 5-6 ft. $\$ 1.00 \quad \$ 10.00$

Mulberry, Tea's Weeping 1.50

Birch, Cut-leaved, 6-8 ft. 1.25

\section{RHODODENDRONS}

Catawbiense Seedlings $\$ 1.50$

Grafted, well budded and with ball of earth

2.00

\section{ORNAMENTAL SHRUBS}

Fine, well rooted Specimen plants

Almond, Flowering, 2-3 ft._-___-_\$ .50 $\$ 4.00$

Althea, single or double, in variety, $2-3 \mathrm{ft}$.

Aralia, Pentaphylla, 3-4 ft._...__ .35

Azalea, Mollis _- 1.00

Barberry, Thunberg's, 2-3 $\mathrm{ft}_{-\ldots} \ldots .40$

Butterfly Bush, heavy roots_______ .35

Calycanthus

Clethra, Alnifolia

Cornus, variegated leaf, $2-3 \mathrm{ft}_{-----} .50$

Cornus, Sibirica, Sericea and Stolonifera, 2-3 ft.

Deutzia, in variety _-

Euonymous, variegated leaf, dwarf_ .35

Exochorda Grandiflora _____ .35

Fringe, Purple and White _._._. .50

Forsythia in variety

Honeysuckle, bush. Pink and White Tartarian, Morrowi, etc. _._._._. .35

Hydrangea P. G., Hardy, bush form, $11 / 2-2$ ft. .

Hydrangea P. G., Hardy, bush form, $2-3$ ft. - .35

Hydrangea P. G., Hardy, tree form 1.00

Hydranga Arborescens Sterilis, the new hardy everblooming sort, begins blooming in July _______ .50

Japan Quince _... 35

Kerria, in variety

Lilac, White and Purple_-_______-_ .35

Lilac, Named, fancy sorts, single and double -

Mahonia Aquifolia _-______. .35

Spirea, Anthony Waterer and Callosa Alba -

Spirea, Von Houtte, 2-3 ft._____. .35

Spirea, Von Houette, 3-4 ft.___._. .40

Spirea, other varieties ________. .35

Syringa, Golden Leaf _.____ .50

Syringa, Bouquet Blanc _-_-_- .50

Syringa, other varieties _-_._-_._. .35

Snowberry, red or white _._.____- .35

Tamarisk _-_. 35

Viburnum, Sterilis, Common Snowball _-

Viburnum, Opulus, High Bush Cranberry _-___._.

Viburnum, Plicatum, Japan Snowball
3.00

3.50

3.00

3.00

3.00

4.00

3.00

$\$ 25.00$

25.00 25.00

3.00

3.00

3.10

4.00

3.00

3.00

2.50

20.00

3.00

7.50

4.00

3.00

3.50

3.00

5.00

3.00

3.50

3.00

3.00

3.00

4.00

4.00

3.00

3.00

3.00

3.00 


$\begin{array}{lrrr} & \text { Each } & \text { Per } 10 & \text { Per } 100 \\ \text { Weigelia, Eva Rathke } & .50 & 4.00 & \\ \text { Weigelia, other varieties } & .35 & 3.00 & \end{array}$

\section{CLIMBING VINES}

Clematis, large flowering -

Clematis, Paniculata --_-_-_-_-_-- .40

$\$ 6.00$

Dutchman's Pipe -..-_- .

Honeysuckle -_-_-_-_-_-_-_-_-_-_ . 35

3.50

6.00

3.00

3.50

Matrimony Vine -

2.50

Wisteria

.35

\section{PAEONIES AND PERENNIAL PLANTS}

See catalogue for prices.

\section{BULBS}

See catalogue for prices.

\section{EVERGREENS-Specimen Trees}

Arbor 'Vitae, 2-3 ft.

Arbor Vitae, 3-4 ft.-_-_-_-_-_-- 1.00

Arbor Vitae, Pyramidal, 2-3 $\mathrm{ft}$

Arbor Vitae, Pyramidal, 3-4 ft.----- 1.00

Fir Balsam, 3-4 ft. -_-_-_-_-_ 1.00

Juniper, Irish and Swedish, 2-3 ft.- $\quad .75$

Pine, Austrian, White and Scotch,

$11 / 2-2$ ft. - -

Pine Austrian, White and Scotch,

2-3 ft. ----

Spruce, Norway, 2-3 ft.

Spruce, Norway, 3-4 ft.

Spruce, Hemlock, 11/2-2 ft..-_..-- 1.00

$\$ 6.00$

We have in stock about thirty varieties of fancy imported evergreens, mostly dwarf growers suitable for use around residences, etc. Some are very rare. List and prices will be sent on application.

We also have the folowing varieties of Yew which are absolutely hardy. The ordinary English and Irish Yew is not hardy here. Of low branching habit:

Taxus (Yew), Cuspidata, 2-3 $\mathrm{ft}_{\text {- }}$

Taxus (Yew), Cuspidata, Brevifolia, $1-1 \frac{1}{2} \mathrm{ft} 1.50$

Box, fine bushy specimens, $12-15$ in.------ 1.00

Box, fine, bushy specimens, 15-18 in..-..-- 1.50

\section{HEDGE PLANTS}

Each Per 10 Per 100

Am. Arbor Vitae, 12-18 in.

$\$ .15 \$ 1.25 \quad \$ 10.00$

Am. Arbor Vitae, 18-24 in._-__-_ .20 $1.75 \quad 15.00$

Althea, seedlings, 2-3 ft.__-__- $.20 \quad 1.50 \quad 12.00$

Thunberg's Barberry, 12-18 in._-- $\quad .20 \quad 1.50 \quad 10.00$

Thunberg's Barberry, 18-24 in.---- $.30 \quad 2.50 \quad 18.00$

Calif. Privet, $12-18$ in. $\quad .10 \quad .50 \quad 4.00$

Calif. Privet, 18-24 in._-___ $.10 \quad .80 \quad 5.00$

Calif. Privet, 2-3 ft., very heavy _- $.15 \quad 1.25 \quad 6.00$

Ibota Privet, more hardy, $12-18$ in.- $\quad .15 \quad .75 \quad 6.00$

Ibota Privet, more hardy, 18-24 in.- $\quad .15 \quad 1.00 \quad 8.00$

Ibota Privet, more hardy, 2-3 ft. $-.20 \quad 1.50 \quad 12.00$

\section{ROSES}

Hybrid Perpetuals and Moss

Krau Karl Druschki, Soliel D'Or,

American Beauty, and Climbing

American Beauty _-_-_-_-_._. $.60 \quad 6.00$

Baby Ramblers - -

Rugosa -

Climbers -

\section{THE FREMONT NURSERY FREMONT, OHIO}

